\title{
Análisis fonológico-grafémico del Vocabulario de Bertonio
}

\author{
Luisa Portilla Durand \\ Departamento Académico de Lingüística
}

IE 1 Vocabvlario aimara del sacerdote Ludovico Bertonio se publicó en

— 1612 y constituyó el más nutrido y útil diccionario de la lengua publicado en ese tiempo. Otro aspecto por el que destaca el Vocabvlario es la buena representación ortográfica de las palabras aimaras, tarea no muy sencilla debido a la complejidad de la fonética de dicha lengua nativa (hay consonantes oclusivas simples, aspiradas y glotalizadas). Si consideramos el limitado saber lingüístico de su época, el trabajo de Bertonio continúa siendo una valiosa y confiable fuente para el léxico y la fonología del idioma altiplánico.

Nos propusimos cỏmpårarta ortografía detBetfonio con la de dos diccionarios aimaras modernos: el Diccionarĭo Aymara de Büttner-Condori (1984) y el Diccionario Aymara-Castellano/ Castellano-Aymara de Manuel de Lucca (1987), recomendado por Xavier Albó en la introducción al Vocabvlario de Bertonio arriba mencionado.

El objetivo de nuestro estudio ha sido verificar el margen de validez de la Tabla Fonémica del Alfabeto de Bertonio, formulada en la edición de su Vocabvlario, y para este fin trabajamos con 377 fichas llenadas cada una con dos entradas de significado similar, difiriendo en que la primera entrada corresponde al aimara usado por Bertonio y la segunda al aimara actual de Büttner-Condori; dichas entradas fueros escogidas al azar del Vocavulario de Bertonio y cotejadas luego con el Diccionario Aymara de Büttner-Condori, cuya ortografía es de base fonológica (considera sólo tres vocales). Terminada 
esta primera parte pasamos a seleccionar los ejemplos para cada fonema y plantear así todas las representaciones grafémicas existentes. Completa esta segunda parte, verificamos si las correspondencias fonémicas planteadas en la edición del Vocabulario de Bertonio funcionan de la misma forma en que ahora se observan.

Seguidamente vamos a desarrollar este último punto, ya que los dos primeros forman parte del trabajo realizado para organizar el material de la presente investigación, el cual consiste -para la primera parte- en 377 fichas en las que se consignan las entradas tomadas como muestra y -para la segunda parte- en un total de 15 hojas adicionales en las que se consignan los fonemas de mayor relevancia para este estudio ${ }^{1}$ y sus diferentes representaciones grafémicas.

FONEMAS, REPRESENTACIONES GRAFÉMICAS E INCONSISTENCIAS

$/ \mathrm{p} /$

Este fonema es representado por Bertonio de dos maneras: $\langle\mathrm{p}\rangle,\langle\mathrm{ph}\rangle$.

$<\mathrm{p}>$

Ejemplos:

232.

(B) Biblioteca, (B-C)

paka Converson aguila'

244. pirca - pirqa

249. puraca - puraka

298. kapitha — q'apiña

'pared'

'barriga'

'apretar'

Inconsistencia $\rightarrow<\mathrm{ph}>$

Esta representación la encontramos en un solo caso:

(B)

292. ccophitha
(B-C)

qhupiña 'tapar...'

1 Cuando decimos que consignamos aquí sólo los fonemas de mayor relevancia, queremos indicar que los otros fonemas no han presentado mayor problema en su representación (de esto, así como de otros aspectos, se trata al final del trabajo). 
$/ \mathrm{p}^{\mathrm{h} /}$

Este fonema es representado por Bertonio de dos maneras: $<\mathrm{ph}\rangle,<\mathrm{p}\rangle$.

$<\mathrm{ph}>$

Ejemplos:

(B)

124. haraphi

253. phako

258. phisca

260. phuccu
(B-C)

- jaraphi 'costillas'

- phaq'u 'rojo...'

- phisqa

'cinco'

'olla'

- phuku

Inconsistencia $\rightarrow\langle p\rangle^{2}$

Ejemplos:

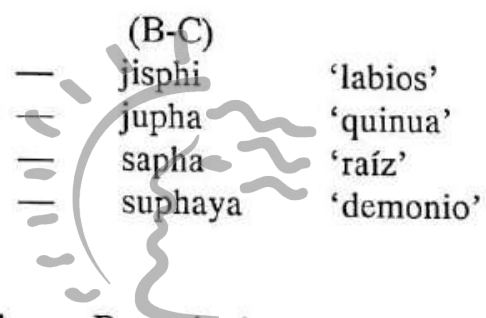

/p'/

142. hispi

151. hupa

307. sapa

317. supayo

'labios'

Este fonema es representado por Bertonio de tres maneras: $\langle p p\rangle,\langle p\rangle,\langle p h\rangle$.

$<\mathrm{pp}>$

Ejemplos:

\section{Biblioteca de Letras}

(B)

133. hayppu

148. humppi

191. lappa

266. ppekeña

269. ppusttatata

\section{"Jorge Puccinelli Converso»}

(B-C)

jayp'u

jump'i

lap'a

p'iqi

p'ust'ata 'tarde'

'sudor'

'piojo'

'cabeza'

'hinchado'

2 Verificando en De Lucca los ejemplos dados para sustentar la inconsistencia, observamos que en el caso de los ejemplos (151) y (307) De Lucca coincide con Büttner-Condori, mientras que para los ejemplos (142) y (317) De Lucca coincide con Bertonio; así para la entrada 'demonio' De Lucca consigna $<$ supaya $>$ con $<p>$ de la misma forma que Bertonio, mientras que Büttner-Condori consignan <suphaya $>$ con <ph>; por último, para la entrada 'labios', Büttner-Condori consignan <ispillu> con $\langle p\rangle$ como sinónimo de $<j i s p h i>$ y al verificar en De Lucca encontramos también $\langle j i s p i\rangle$ con $\langle p\rangle$. Esto indicaría que existen sólo dos ejemplos de inconsistencia de $/ \mathrm{p}^{\mathrm{h} /} / \mathrm{como}\langle\mathrm{p}\rangle$. 
Inconsistencia $\rightarrow\langle\mathrm{p}\rangle^{3}$

Ejemplos:

(B)

(B-C)

199. lirpu

- lirp'u

264. pequitha - p'akiña

'espejo'

203. Ilumpaca - llump'aqa

'quebrar'

311. sillpi

- sillp'i

'limpio'

'cascara o corteza'

Inconsistencia $\rightarrow\langle\mathrm{ph}\rangle$.

Esta representación la encontramos en un solo caso:

(B)

268. phukhru
(B-C)

- p'ujru 'hondo'

/t/

Este fonema es representado por Bertonio de la misma forma, con $\langle t\rangle$, pero hay un caso en el cual se representa con $\langle\mathrm{t}\rangle$ :

(B)

118. hamppattitha - jamp'atiña 'besar'

$\mid \mathrm{c}^{\mathrm{h}} /$

Este fonema es represēntado poe Bertoniō de tres maneras: <chh>, <cch>, $<\mathrm{ch}>$.

$<$ chh>

Ejemplos:

(B)

56. chhaccatha -

61. chhokhri -
(B-C)

chhaqayaña 'perder (se)'

chhuxri 'herida...'

3 Esta inconsistencia parece corroborarse, ya que al verificar en De Lucca encontramos que su representación coincide con la de Büttner-Condori para los ejemplos (264) y (311). Lamentablemente, para los ejemplos (199) y (203) De Lucca no consigna entradas. 
Inconsistencia $\rightarrow\langle\mathrm{cch}\rangle^{4}$

(B)

57.

59.

cchakhrutha

60.
(B-C)

chharquña 'mezclar'

chhipchhi

chhukuña 'pestañear/pestaña'

'correr en grupo'

\section{Inconsistencia $\rightarrow\langle\mathrm{ch}\rangle^{5}$}

(B)

8. allchi
(B-C)

allchhi

chhijchhi 'nieto(a)'

'granizo'

|č'|

Este fonema es representado por Bertonio de tres maneras: <cch $>,<\mathrm{chh}>$, $<\mathrm{ch}>$.

$<$ cch $>$

Ejemplos:

57. cchaca

76. cchin̄i

84. cchuñu

107. haccha

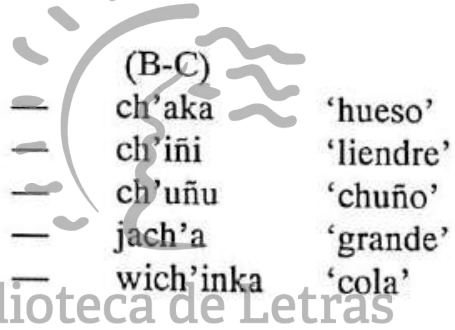

Inconsistencia $\rightarrow<$ chlisge Puccinelli Converso»

Esta representación la encontramos en un solo caso:

4 De los tres ejemplos presentados para plantear la inconsistencia dada, verificamos en De Lucca que los ejemplos (57) y (60) son representados de la misma forma que BüttnerCondori, pero no así el ejemplo (59) donde De Lucca presenta para 'pestañear' <ch'ipeqeña>, con $/ \breve{c} /$, lo que coincidiría con la representación de Bertonio <cchiphikhtatha>, ya que para él $<c c h\rangle$ representa a /č'/ (caso que veremos posteriormente). De esta manera podría quedar descartado el ejemplo (59) como prueba de inconsistencia.

5. Al revisar en De Lucca los ejemplos planteados para sustentar la inconsistencia pudimos confirmar que éste coincide plenamente con la representación de Büttner-Condori, pero al revisar nuevamente el vocabulario de Bertonio encontramos -para el caso del ejemplo (58)que consigna la entrada 'granizar' como <chhikhchhitha> transcripción que coincide con la de Büttner-Condori y con la de De Lucca, con la diferencia de que estos consignan 'granizo', pero en suma la raíz es la misma; de esta manera, la inconsistencia nula. 
(B)

(B-C)

82. chhukhlla - ch'ujlla 'choza'

Inconsistencia $\rightarrow\langle\mathrm{ch}\rangle^{6}$

Ejemplos:

(B)

84. chullchu

226. ñachatha
(B-C)

ch'ullch'u 'flaco...'

ñach'aña 'amarrar...'

\section{/k/}

Este fonema es representado por Bertonio de cuatro maneras: $\langle\mathrm{c}\rangle$, $\langle\mathrm{qu}\rangle$, $<\mathrm{cc}\rangle,\langle\mathrm{qhu}\rangle$.

$<\mathrm{c}>/-\mathrm{a}, \mathrm{u}^{7}$

Ejemplos:

(B)

20. arcatha

37. chacu

155. callachi

165. cunca

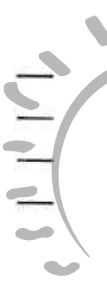

(B-C)

arkaña 'seguir'

chaku $\sim$ 'caza'

kallachin 'hombro'

kunka

'cuello, garganta'

$<q u>/-$ i

Ejemplos:

25.

(B)

Bibliotecq $\mathrm{d}-\mathrm{C})$
"Jorge Pucciaski.

Ietras

28. iquitha - ikiña

159. quimsa - kimsa

'dormir'

'tres'

6 De los ejemplos presentados para esta inconsistencia, cl ejemplo (226) es representado por De Lucca de la misma forma que Büttner-Condori, pero en lo que respecta al ejemplo (84) encontramos que De Lucca consigna 'flaco' como <chullchu>, sin $/ \bar{c}$ ' $/$, a diferencia de Büttner-Condori, que consignan la misma entrada <ch'ullch'u> con /č'/ en las dos sílabas; en este sentido, entonces, De Lucca coincide con la representación de Bertonio, que también consigna <chullchu>. De esta forma el ejemplo (84) podría no ser una inconsistencia.

7 Aquí se presenta una inconsistencia, ya que <c> representando al fonema $/ \mathrm{k} /$ también se presenta delante de "o" lo que hace que se confunda con la representación del fonema / q/ que delante de "o" se representa con $<c>$. Veamos:

(B)

163.

243.

\section{(B)}

(B-C)

kullaca

pinkillu 'hermana'

'flauta' 
Inconsistencia $\rightarrow\langle\mathrm{cc}>/-\mathrm{u}$

Ejemplos:

(B)

64.

260. $\begin{array}{ll}\text { cchaccutha } & - \\ \text { phuccu }^{8} & -\end{array}$
(B-C)

ch'aku

phuku 'darle un moxicón/puñete...'

'olla'

$\mathrm{La}$ inconsistencia se basa en que la grafía $<\mathrm{cc}>$ representa al fonema /

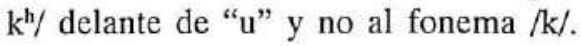

La inconsistencia $\vartheta<q h u>/-i$

Esta representación la encontramos en un solo caso:

(B)

70. ccharqhui $^{9} \quad$ h'arki 'cecina/carne seca'

La inconsistencia se basa en que la grafía <qhu> representa al fonema $/ \mathrm{k}^{\mathrm{h}} /$ delante de "i" y no al fonema $/ \mathrm{k} /$,

$/ \mathrm{k}^{\mathrm{h}} /$

Este fonema es representado por Bertonio de tres maneras: $\langle\mathrm{cc}\rangle,\langle\mathrm{qhu}\rangle,\langle\mathrm{k}\rangle$. $<\mathrm{cc}\rangle$ / - a, u

8 De los dos ejemplos presentados para la inconsistencia planteada, el ejemplo (260) tiene cierto margen de descarte. Vecmos pof que,-de acuerdo âas' diferentes representaciones:

$\begin{array}{ll}\text { Bertonio } & \rightarrow \text { phuccu 'olla', phucuyana 'olla toda negra' } \\ \text { Büttner-Condori } & \rightarrow \text { phuku 'olla' } \\ \text { De Lucca } & \rightarrow \text { phukhu 'olla' }\end{array}$

1. Si cotejamos <phuccu> con <phukhu> observamos que la representación de Bertonio coincide con la de De Lucca, ya que para Bertonio la grafía <cc> representa al fonema $/ \mathrm{k} /$ delante de " $\mathrm{u}$ ".

2. Ahora bien, pero ¿qué pasa con <phucuyana> y <phuku>? Si bien en el primer caso el nombre es modificado por un adjetivo, la raíz nominal representa en, ambos casos, a la misma entrada 'olla'. De esta manera Bertonio coincidiría con Büttner-Condori, ya que para Bertonio <c> representa al fonema $/ \mathrm{k} /$ delante de " $\mathrm{u}$ ".

9 Para el ejemplo (70), De Lucca tiene dos representaciones: <ch'arki> y <ch'arkhi> 'carne seca'. Ahora bien, si Bertonio, que consigna la misma entrada como <ccharqhui>, no coincide en su representación con Büttner-Condori que presentan <ch'arki>, sí coincide con la segunda representación de De Lucca, ya que para Bertonio la grafía <qhu> representa al fonema $/ \mathrm{k}^{\mathrm{h}} /$ delante de "i". De esta manera el ejemplo (70) podría no ser considerado una inconsistencia. 
Ejemplos:

(B)

108.

136.

140

178. ccuchi
(B-C)

\section{khakha}

jikhani

jiskhu

khuchi 'tartamudo'

'espalda'

'ojotas'

'puerco'

<qhu>/ - i

Ejemplos:

(B)

139.

175. hisqh

qhuistutha
-
(B-C)

jiskhiña

khistuña 'preguntar'

'mascar'

Inconsistencia $\rightarrow<\mathrm{k}>/-\mathrm{a}$

Esta representación la encontramos en un solo caso:

170. kanasitha ${ }^{10}$ - khanuña 'rumiar'

La inconsistencia se basa en que la grafía <k> representa al fonema / $\mathrm{k}$ '/ o /q"/ delante de "a" y no al fonema $/ \mathrm{k}^{\mathrm{h}} /$.

$/ \mathrm{k}^{\prime} /$

\section{Biblioteca de Letras}

Este fonema es representado por Bertonio de dos maneras: $\langle\mathrm{k}\rangle,\langle\mathrm{c}\rangle$. $<\mathrm{k}>/-\mathrm{a}, \mathrm{i}, \mathrm{u}^{11}$

10 Respecto a la inconsistencia planteada podríamos decir, a partir de la verificación hecha en De Lucca, que no es válida, ya que este autor nos da dos representaciones para la entrada 'rumiar': <khanuña>, que coincide con la representación de Büttner-Condori, y <k'anuña>, que coincide con la de Bertonio, quien consigna $<$ kanasitha $>$, con $<k>$, que representa al fonema $/ \mathrm{k} \%$, el cual también representa De Lucca.

$11<\mathrm{k}>$ también se presenta delante de "o". Veamos:

De Lucca

$$
\begin{aligned}
& \text { 229. ñakota - ñik'uta - ñik'uta, ñak'uta 'cabello' } \\
& \text { 359. huanko - wank'u - wank'u 'cuy' }
\end{aligned}
$$

Estas representaciones son muestra de inconsistencia, ya que $<k>$ delante de "o" representa al fonema $/ q$ '/ y no al fonema $/ k^{\prime} \%$. 
Ejemplos:

(B)

(B-C)

141. hiska

jisk'a

'pequeño'

183. kara

k'ara

'pelado'

187. killima

k'illima

'carbón'

189. kumu

k'umu

'jorobado'

197. liki

lik'i

'gordura'

Inconsistencia $\rightarrow<\mathrm{c}>/-\mathrm{u}^{12}$

Esta representación la encontramos en un solo caso:

(B)

(B-C)

365. huaycutha - wayk'uña 'teñir'

La inconsistencia se basa en que la grafía $<c>$ representa al fonema / $\mathrm{k} /$ delante de «u» y no al fonema $/ \mathrm{k} \%$.

$/ \mathrm{q} /$

Este fonema es representado por Bertonio de cuatro maneras: $<\mathrm{c}\rangle,<q u>$, $<\mathrm{cc}\rangle,\langle\mathrm{k}\rangle$.

$<\mathrm{c}>1-\mathrm{a}, \mathrm{o}$

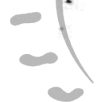

Ejemplos:

16. anocara Jorge Puccianucharanverserro'

205. lloco - lluqu 'corazón'

275. carcutha ${ }^{13}$ - qarquña 'desterrar'

279. collatha - qullaña 'cerrar'

12 Lamentablemente el ejemplo para esta inconsistencia no pudo ser verificado en De Lucca, ya que no consigna esta entrada; por lo tanto, la inconsistencia se mantiene.

13 En este ejemplo se puede observar que la grafía $\langle c\rangle$ se presenta delante de "u", lo cual es una inconsistencia, ya que $\langle c\rangle$ delante de " $u$ " representa al fonema $/ \mathrm{k} /$ y no al fonema /q/. Esto hace notar que existe una confusión al tratar de representar los fonemas $/ \mathrm{k} / \mathrm{y} /$ $q /$, porque hay casos en los cuales la grafía $<c>$ pretendiendo representar al fonema $/ q /$ se presenta en ambientes que corresponden al fonema $/ \mathrm{k} /$, y hay otros casos en los cuales la grafía $<\mathrm{c}>$ pretendiendo representar al fonema $/ \mathrm{k} /$ se presenta en ambientes que corresponden al fonema /q/ (ver nota 7). Ahora bien, lo señalado no invalida nuestro ejemplo, pues lo que interesa aquí es el uso de la grafía $<\mathrm{c}>$ delante de "a" re - centando al fonema / $\mathrm{q} /$. 
$<q u>/-e$

Ejemplos:

(B)

(B-C)

87. cchoque - ch'uqi

121. heque - jaqi

276. quelo - qilu

278. quenaya - qinaya

'papa...'

'hombre...'

'cojo'

'nube'

Inconsistencia $\rightarrow\langle\mathrm{cc}\rangle /-\mathrm{a}, \mathrm{o}^{14}$

Ejemplos:

(B)

(B-C)

18. acco

aqu

'molar'

56. chhaccatha - chhaqayaña 'perder'

69. cchaccatha - ch'aqaña 'gotear'

330. thaccatha - thaqaña 'buscar'

349. occara

uqara 'sordo'

La inconsistencia se basa en que la grafía <cc> representa a los fonemas $/ \mathrm{k}$ h/ o /q" delante de "a", "u" o "a" "o", respectivamente, pero no al fonema $\mathrm{qq} /$.

Inconsistencia $\rightarrow<$ Biloffoteca de Letras

Esta representación la la eneontramos en un solo caso:

14 Sobre los ejemplos presentados para sustentar la inconsistencia planteada debemos aclarar algunos aspectos:

1..$^{2}$ Los ejemplos (56) y (330) podrían no ser casos de inconsistencia, ya que al verificar dichas entradas de De Lucca pudimos constatar que sus representaciones no coinciden con las de Büttner-Condori, pues De Lucca consigna los verbos 'buscar' y 'perder' como <thaqhaña> $y<$ chhaqhaña>, respectivamente -ambos con $/ q^{\mathrm{h}} /-$, a diferencia de Büttner-Condori, que consignan <thaqaña> y <chhaqayaña >-con $/ q /-$, lo que indicaría que la representación de Bertonio coincide con la de De Lucca, porque la grafía $\langle c c\rangle$ representa para Bertonio al fonema $/ \mathrm{q}^{\mathrm{h}} /$ delante de "a". De esta manera dichos ejemplos no serían casos de inconsistencia.

2.9 En lo que respecta a los ejemplos (18), (69) y (349), debemos indicar que la inconsistencia se mantiene, pues al verificar en De Lucca encontramos que sus representaciones coinciden con las de Büttner-Condori, oponiéndose así a las representaciones de Bertonio. 
(B)

(B-C)

266. ppekeña ${ }^{15}$ - p'iqii 'cabeza'

La inconsistencia se basa en que la grafía $<\mathrm{k}>$ representa al fonema / q'/ delante de "e" y no al fonema /q/.

$/ \mathrm{q}^{\mathrm{h}} /$

Este fonema es representado por Bertonio de tres maneras: $\langle\mathrm{cc}\rangle,\langle\mathrm{qhu}\rangle,\langle\mathrm{c}\rangle$. $<\mathrm{cc}>1-\mathrm{a}, \mathrm{o}^{16}$

Ejemplos:

(B)

286

136. cconath

140. ccophith

$<$ qhu $>$ / - e

Ejemplos:

(B)

122. haqhue

200. loqhue

285. qhuella
(B-C)

qhultuña 'bramar'

qhunaña 'restregar'

qhupiña 'tapar...'

Inconsistencia $\rightarrow<c>$ Biblioteca de Letras

Esta representación la encontramos en un solo caso:

367. huaycu' ${ }^{17} \quad$ wayqhu, wayq'u 'quebrada'

15 Al verificar en De Lucca el ejemplo mostrado para sustentar la inconsistencia, encontramos que consigna <p'eqeña> 'mente' y <p'eqe > 'cabeza', de manera tal que ambas entradas se asemejan a la transcripción de Büttner-Condori y no a la de Bertonio, de lo cual concluimos que el ejemplo mostrado sí es un caso de inconsistencia.

16 Cabe indicar aquí, que si bien en ninguno de los ejemplos planteados se consigna la grafía $<c c>$ seguida de "a", por simetría dedujimos su presencia en dicho ambiente.

17 En este caso pudimos ver, al revisar el vocabulario de De Lucca, que la representación que consigna no coincide ni con Büttner-Condori ni con Bertonio -aunque tal vez en algo con este último-, ya que para la entrada 'quebrada' consigna <wayk'u> con $/ k$ '/, mientras que Büttner-Condori consigna <wayqhu> y <wayq'u>con /qh/ y /q'/, respectivamente, y Bertonio $<$ huaycu>; de lo que sólo podríamos decir que este último debió haber captado mal el 
$\mathrm{La}$ inconsistencia se basa en que la grafía $<\mathrm{c}>$ delante de "u" representa al fenema $/ \mathrm{k} /$ y no al fonema $/ \mathrm{q} /$.

'q']

Este fonema es representạdo por Bertonio de dos maneras: $<\mathrm{k}>,<\mathrm{cc}>$.

$<\mathrm{k}>\mathrm{l} \rightarrow \mathrm{a}, \mathrm{e}, \mathrm{o}$

Ejemplos:

(B)

(B-C)

192. laka

laq'a

'tierra'

230. ñeke

ñiq'i

'barro'

253. phako

- phaq'u

'rojo, vermejo'

296. kañu

'sucio'

300. kello

- q'illu

'amarillo'

303. koma

- q'uma

'limpio'

Inconsistencia $\rightarrow<\mathrm{cc}>/-\mathrm{u}$

Esta representación la encontramos en un solo caso:

(B)

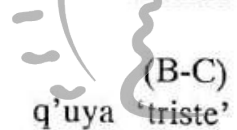

304. ccuya ${ }^{18}$ - q'uya triste'

$\mathrm{La}$ inconsistencia se basa en que la grafia $\langle\mathrm{Cc}\rangle$ delante de «u» representa al fonema $/ \mathrm{k}^{\mathrm{k}}$ / y hegaf forfenalli $\mathrm{q} \%$.

/j/

Este fonema es representado por Bertonio de dos maneras: $<\mathrm{h}>,<\mathrm{kh}>$. $<\mathrm{h}>\int_{\mathrm{Va}-\mathrm{Va} .}^{\#-\mathrm{a}, \mathrm{i}, \mathrm{u}}$

sonido velar glotal / k'/ de De Lucca, confundiéndolo con una velar simple $/ \mathrm{k} /$ por lo que la representa con $\langle c\rangle$.

18 Al parecer nos encontramos frente a un caso de captación acústica defectuosa, pues pareciera que Bertonio escuchó una velar y no una postvelar; por ello representa con la grafía $<c c>$ el fonema velar $/ \mathrm{k}^{\mathrm{h} /}$ que creyó escuchar. 
Ejemplos:

(B)

4. ahano

106. hachatha

137. hilatha

146. hucha
(B-C)

ajanu

jachaña

jilaña

jucha 'cara'

'llorar'

'crecer'

'culpa'

$<k h>/ \begin{aligned} & \text { s/ }-\mathrm{a}, \mathrm{i}, \mathrm{u}^{*} \\ & \mathrm{a}, \mathrm{i}, \mathrm{u}^{*}-\mathrm{s}\end{aligned}$

Ejemplos:

(B)

42. chikhi

58. chhikhch

62. cchakha

356. huakhcha.
(B-C)<smiles>[SiH2]=[GeH2]</smiles>

'desgracia'

'granizo'

'ronco'

'huérfano...' chhijchhi

ch'aja

wajcha

\section{* "u" se deduce por simetría.}

/x/

Este fonema es representado por Bertonio con la grafía $<\mathrm{kh}>$ en distribución complementaria (DC) con el caso anterior ${ }^{19}$.

\section{Biblioteca de Letras}

$<\mathrm{kh}>/ \begin{aligned} & \mathrm{s} /-\mathrm{a}^{*}, \mathrm{e}, \mathrm{o}^{*} \text { «Jorge Puccinelli Converso» } \\ & \mathrm{a}, \mathrm{e}^{*}, \mathrm{o}-\mathrm{s}\end{aligned}$

19 La DC explica la presencia de la misma grafía <kh>, tanto para representar al fonema / $\mathrm{j} /$ como al fonema $/ \mathrm{x} /$, por lo que en el primer caso nos encontramos frente a una fricativa velar y en el segundo caso frente a una fricativa postvelar, en contacto siempre con las vocales medias "e", "o". La única dificultad que podría presentarse para deslindar a qué fonema corresponde la grafia <kh>, sería frente a la vocal "a". (El mismo caso podemos observar cuando se representan los fonemas $/ \mathrm{k} /-/ \mathrm{q} /, / \mathrm{k}^{\mathrm{h}} /-/ \mathrm{q}^{\mathrm{h}} /$ y $/ \mathrm{k} /-/ \mathrm{q}^{\prime} /$ delante de "a", donde la grafía para cada par de fonemas es la misma frente a esta vocal.) 
Ejemplos:

(B)

72.

74. 89.

(B-C)

ch'axiña 'machucar'

ch'axmiña 'molestar'

ch'uxña 'verde'

* "a", "o", "e" se deducen por simetría.

/w/

Este fonema es representado por Bertonio de dos maneras: <hu $>,<\mathrm{u}\rangle$.

$<$ hu>l - a.

Ejemplos:

144. hihuatha - jiwaña 'morir'

194. lahua - lawa 'palo'

360. huaña - waña 'seco'

361. huararitha - wararina - 'gritar...'

368. huayu wayu 'racimo'

$<\mathrm{u}>\quad \begin{gathered}\mathrm{a}, \mathrm{i}-- \\ -\mathrm{i}, \mathrm{u}\end{gathered}$

Ejemplos:

28. (B) Biblioteca-de Ietras

130. hauira - jawira 'rio'

145. hiuke - jiwq'i 'humo'

160. quiuu - kiwu 'colmillo'

176. qhuiuitha - khiwiña 'enrrollar...'

370. uila - wila 'sangre'

\section{CONCLUSIONES}

Para comenzar debemos aclarar aquí algo más sobre la llamada 1, esto es, el hecho de no haber tratado en el presente trabajo el estudio de todas las grafías que se consignaron en las fichas, y los respectivos fonemas que éstas representan. La razón de esta «omisión voluntaria» se debe -como se dijo al iniciar este trabajo- a que no muestran mayores diferencias respecto a su 
representación por parte de Bertonio y Büttner-Condori, éste es el caso de los fonemas $/ \mathrm{a} /, / \check{\mathrm{c}} /, / \mathrm{i} /, / \mathrm{I} /, / \mathrm{I} /, / \mathrm{m} /, / \mathrm{n} /, / \tilde{\mathrm{n}} /, / \mathrm{r} /, / \mathrm{s} /, / \mathrm{t} /, / \mathrm{t}^{\mathrm{h}} /, / \mathrm{t} /, / \mathrm{u} /, / \mathrm{y} /$, cuyas representaciones sí fueron consideradas en las 377 fichas que constituyeron la primera parte del material que fue analizado para realizar esta investigación. Los fonemas que sí mostraron variaciones en su representación -además de encontrarse en las fichas respectivas- se consignaron en 15 hojas adicionales que constituyeron la segunda parte del material que fue analizado para realizar este estudio.

Respecto a la validez de la Tabla Fonémica planteada para el Alfabeto de Bertonio, debemos decir que ésta funciona muy regularmente, aunque con ciertas fallas que vamos a citar ahora:

En primer lugar, y de manera general, diremos que no se hacen las salvedades correspondientes en los casos en que los fonemas son representados por más de una grafía. Se da por sentado que a cada fonema corresponde una grafía o a lo más dos-de acuerdo a los ambientes-, como es el caso de velares y postvelares. Esto se observa prácticamente con todos los fonemas que hemos estudiado aquí, demostrándose a través de las inconsistencias planteadas que no a todo fonema corresponde sólo una representación grafémica; hay también casos donde se entrecruzan las mismas grafías representando a fonemas distintos. Se presenta un solo caso en el cual se hace una observación y es respecto al fonema postvelar tq eys dice que "ocasionalmente /q/ aparece comp cc" lo que no es completamente cierto, pues en la pequeña muestra con que hemos trabajado $-\mathrm{y}$ sólo eligiendo entradas al azarencontramos cinco casos donde /q/ aparece como $\langle\mathrm{cc}>$, lo cual indicaría que este hecho no es tan ocasional, lo ocasional sería tal vez que /q/ apareciera como $<\mathrm{k}>$, ya que en nuestro corpus encontramos sólo un caso.

En segundo lugar -y agregando algo más a lo mencionado anteriormente- diremos que los fonemas fricativos $/ \mathrm{j} / \mathrm{y} / \mathrm{x} /$ no han sido tratados con la precisión necesaria, ya que en la Tabla Fonémica se plantea para el fonema /j/ las representaciones grafémicas $\langle\mathrm{h}\rangle,\langle\mathrm{g}\rangle \mathrm{y}\langle\mathrm{gh}\rangle$, mientras que por nuestra parte - tal vez por el tamaño de nuestro corpus - no llegamos a obtener las grafías $<$ g $>$ y $<$ gh $>$, pero $s i ́<$ h $>$ y $<$ kh $>$; esta última grafía en $D C$ representa tanto al fonema velar /j/ como al fonema postvelar / $\mathrm{x} /$ (caso que puede comprobarse en el acápite correspondiente), permitiéndonos así establecer que el fonema $/ \mathrm{j} /$ se representa a través de las grafías $\langle\mathrm{h}\rangle \mathrm{y}\langle\mathrm{kh}\rangle$, y el fonema /x/ a través de $<\mathrm{kh}>$ en DC con su similar. 
En tercer lugar, respecto al fonema semiconsonántico /w/, cabe señalar que nosotros no encontramos este fonema en contacto con las vocales medias [e] y [o], y en cambio sí en contacto con las vocales altas [i], [u]. Sería muy útil conocer los ejemplos que sustentaron tal planteamiento.

Para terminar, quisiéramos destacar aquí la precisión con que maneja Bertonio sus transcripciones; a él no podemos reclamarle nada. Errores tiene, pero considerando que no era fonetista y mucho menos lingüista establece una relación por de más regular entre las grafías y los fonemas que él implícitamente representa. Las inconsistencias las hemos señalado en su momento y son a su vez evidentemente comprensibles; éste es el caso de velares y postvelares en cuyas representaciones las fallas son mínimas, más bien son relativamente mayores en el caso de la oclusiva bilabial y la africada. A nuestro parecer, los errores de transcripción se deben a problemas de captación acústica, los cuales todos podemos comprender; existen así errores de representación en el caso de los fonemas aspirados y glotalizados, pero éstos son pocos, tanto es así que de las inconsistencias que se plantearon muchas de ellas dejaron de ser tales al volverse a verificar. Ahora bien, volviendo por última vez a los errores de representación de los fonemas velares y postvelares, en los cuales observamos casos de entrecruzamiento de las grafías, esto debe entenderse como una manifestación propia de quien no ha tenido una adecuada preparación fonética, de quien ve frente a sí una lengua que tiene sonidos completamente diferentès å los de da suya y que aun así trata de establecer correspondencias grafémico-fonéticas bastante regulares, y casi lo logra.

\section{BIBLIOGRAFÍA}

BÜTTNER, Thomas y CONDORI, Dionisio

1984 Diccionario Aymara-Castellano, Puno, Proyecto Experimental de Educación Bilingüe.

BERTONIO, Ludovico

[1612] 1984 Vocabulario de la lengua aymara. Edición Facsimilar basada en la de 1956. Cochabamba, CERES.

DE LUCCA D., Manuel F. 1987

Diccionario Práctico Aymara-Castellano/Castellano-Aymara, Bolivia, Los Amigos del Libro. 